\title{
Lo emocional como articulador de la razonabilidad en la didáctica de la matemática*
}

\author{
The emotional as an articulator of reasonability \\ in the mathematics didactics
}

Rubén Darío Henao Ciro*** y Mónica Moreno Torres****

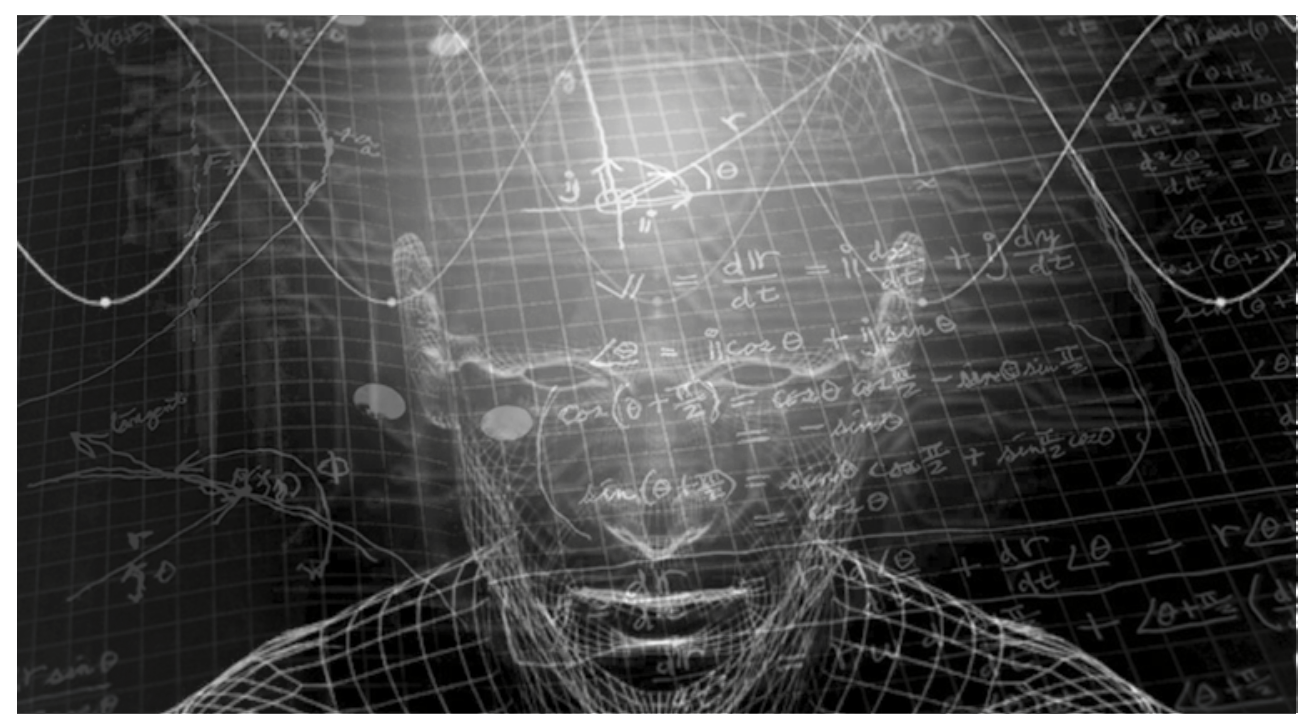

\section{Resumen}

El texto presenta una revisión crítica de la matemática emocional, al tiempo que trata el papel de las emociones en la reconfiguración de la didáctica de las matemáticas en relación con la razonabilidad ${ }^{1}$. Durante mucho tiempo, la racionalidad se ha impuesto sobre la razonabilidad, de tal suerte que la matemática es vista como un área dura que

Citar este artículo como: Henao, R. y Moreno, M. (2016). Lo emocional como articulador de la razonabilidad en la didáctica de la matemática. Revista Papeles, 8(16), 26-34.

Fecha de recibido: marzo 15 de 2016.

Fecha de aceptación: junio 15 de 2016.

* Este documento surge en el marco del doctorado en la línea Didáctica de la Educación Superior, Universidad de Antioquia, cuya tesis es la relación entre textos literarios y científicos como medio para desarrollar la razonabilidad de profesores de matemáticas: una estrategia didáctica en la educación y superior.

*: Profesor de cátedra de la Licenciatura en Matemática y Física de la Facultad de Educación de la Universidad de Antioquia, y docente de la IE Normal Superior de Medellín. Candidato a doctor en educación superior. Correo: rdhenao55@gmail.com

**:* Profesora de la Facultad de Educación de la Universidad de Antioquia. Doctora en Educación, Línea Didáctica Universitaria, de la Facultad de Educación de la misma universidad. Correo: monica.moreno@udea.edu.co

1 En el marco de la mencionada tesis doctoral se ha reconfigurado el concepto de razonabilidad como un proceso interhumano y abductivo que le permite a una persona establecer nuevas conexiones dialógicas desde lo que es, lo que siente, lo que lee y lo que piensa. En este proceso, el ser capta el mundo a través de los sentidos, la imaginación y la razón, y se muestra más razonable en sus acciones. 
causa displacer y sufrimiento a los adolescentes y a los jóvenes. No obstante, en los últimos años han surgido varias investigaciones tendientes a vincular lo cognitivo con lo afectivo y pensar que una educación emocional, paralela a la educación matemática, contribuye a elevar no solo el nivel de conocimientos sino también la calidad de vida de quienes estudian.

Palabras clave: matemática emocional, actitudes, efectos, creencias, didáctica de la matemática.

\begin{abstract}
This text presents a critical review of emotional mathematics while addressing the role of emotions in the reconfiguration of mathematics didactics in relation to reasonableness. For a long time rationality has been imposed on reasonableness, so that mathematics is seen as a hard area that causes displeasure and suffering to adolescents and young people. However, in the last few years a number of researches have emerged to link the cognitive with the affective and to think that an emotional education, parallel to mathematical education, contributes to raise not only the level of knowledge but also the quality of life of those who study.
\end{abstract}

Keywords: emotional mathematics, attitudes, purposes, beliefs, teaching mathematics.

\title{
Introducción
}

Muchas investigaciones demuestran que el aprendizaje de la matemática se ve disminuido o aumentado debido a las emociones y actitudes de estudiantes y profesores (McLeod, 1994; Blanco \& Guerrero, 2002; Bueno, Teruel, \& Valero, 2005; Gil et al., 2006, Gómez Chacón, 2000, 2003, 2009); todos coinciden en que las emociones forman parte de la enseñanza de las matemáticas, puesto que es evidente la relación entre lo emocional y lo cognitivo como un todo dialéctico (Estrada \& Diez, 2011; García, 2012), dado que "el aprendizaje está determinado por la dimensión racional y la dimensión afectiva nos adentra de lleno en la compleja relación entre cognición-afectividad, razón-emoción, mente-corazón" (Bueno et al., 2005, p. 170).

Una de estas investigaciones es la obra Matemática emocional; los afectos en el aprendizaje matemático, donde Gómez (2000) establece una relación entre lo emocional y la matemática escolar. Para la autora, la dimensión afectiva en matemáticas es un "extenso rango de sentimientos y humores que son generalmente considerados como algo diferentes de la pura cognición" (p. 22), apoyada en descriptores como las creencias, las actitudes, los valores y las apreciaciones. Estos descriptores afectan también la educación de personas adultas (Estrada y Diez, 2011).

Bazán y Aparicio (2006) integran los componentes anteriores al conativo, porque consideran que dicha integración provoca una mayor compresión de la matemática. Bueno et al. (2005) aportan el estudio de las competencias socioafectivas en la formación inicial de maestros. Bisquerra (2005) plantea que la finalidad de la educación es el desarrollo humano y en esto es fundamental la formación del profesorado a través de la educación emocional al desarrollarse competencias como: la conciencia emocional, la regulación de emociones y la motivación. Teruel (2000) considera la necesidad de incluir la educación emocional en el currículo, puesto que en la escuela se deben desarrollar "cualidades emocionales, 
como la capacidad de adaptación, las habilidades sociales, la empatía, el autocontrol, la autoconsciencia o conocimiento de sí mismo, el entusiasmo, la automotivación personal, la asertividad, la perseverancia, la amabilidad, el respeto" (p. 142). Fernández (2005) propone el modelo Personalidad y Relaciones Humanas, creado por André Rochais en 1979, para la formación de educadores, el cual interrelaciona "lo que pensamos, lo que sentimos, lo que somos de fondo, el cuerpo y, finalmente, la conciencia profunda" (Fernández, 2005, p. 247). Extremera y Fernández-Berrocal (2003) se refieren al conocimiento emocional como "la capacidad para comprender las emociones junto con una mayor comprensión de las conexiones entre pensamientos y sentimientos, sus determinantes y sus consecuencias" (p. 102). Hidalgo et al. (2008) realizaron un estudio longitudinal del componente emocional matemático y demostraron que hay un fuerte descenso en las actitudes positivas hacia la matemática en estudiantes al pasar de los 11 a los 15 años.

Estas investigaciones se apoyan en trabajos previos sobre las inteligencias, tanto de Daniel Goleman como de Howard Gardner, donde la inteligencia emocional es entendida como

una destreza que permite conocer y manejar nuestros propios sentimientos, interpretar o enfrentar los sentimientos de los demás, sentirse satisfechos y ser eficaces en la vida a la vez que crear hábitos mentales que favorezcan nuestra propia productividad (Goleman, 1995, p. 57).

Así mismo, el autor de las inteligencias múltiples dice que "si queremos que los estudiantes lleguen a aprender, dominar y aplicar algo con criterio, debemos procurar envolver ese algo en un contexto que haga intervenir las emociones" (Gardner, 2000, p. 89); esto es, la educación matemática necesita relacionar otros procesos intrapersonales e interpersonales que nos definen como seres dialécticos y maduros, con pleno control de nuestras

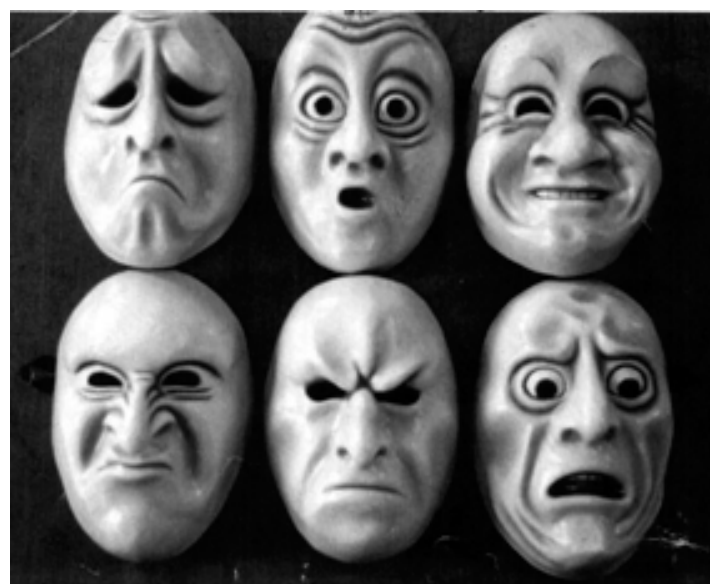

emociones y afectos. McLeod (1994) define el afecto como "un extenso conjunto de sentimientos y humores (estado de ánimo) que son generalmente considerados como algo diferente de la pura cognición".

Phillip (2007) se refiere a la educación matemática afectiva "como el conjunto de maneras de actuar, sentir o pensar que muestran la disposición o la opinión de una persona" (citado por Estrada y Diez, 2011, p. 118), y dice que las actitudes ${ }^{2}$, cognitivas emocionales, se relacionan con sentimientos como resultado de experiencias anteriores; las actitudes negativas se relacionan con el bajo rendimiento en matemáticas (Bazán et al., 2001).

\footnotetext{
Se observa una sinonimia entre afecto, emoción y sentimiento y cómo estos dependen de las actitudes que tengan las personas. La actitud es definida por Hart (1989) como "una predisposición evaluativa (positiva o negativa) que determina las intenciones personales e influye en el comportamiento" (citado en Caballero \& Blanco, 2007, p. 4); las actitudes son "predisposiciones aprendidas para responder positiva o negativamente a objetos dados, situaciones, conceptos o personas" (McLeod, 1992, citado por Estrada \& Diez, 2011, p. 118). Para Gal et al (1997) las actitudes son la "suma de emociones y sentimientos que se experimentan durante el período del aprendizaje de la asignatura objeto de estudio" (Bazán \& Aparicio, 2006, p. 5). Más todavía, las actitudes "son manifestaciones de la conducta que tienen su origen en creencias, emociones, hábitos y experiencias anteriores" (Castelló et al., 2010, p. 66).
} 
Además, Vigotsky (2004) dice que el pensamiento es posible si se comprende la base afectiva y volitiva de la persona. Los afectos ayudan en la estructuración del pensamiento. Según Spinoza, "los afectos son estados del cuerpo que aumentan o disminuyen la capacidad de éste para la acción, que favorecen dicha capacidad o la limitan pudiendo favorecer o no la consciencia de esos estados" (citado por Vigotsky, 2004, p. 4).

Vigotsky, en un ensayo sicológico publicado en 1982 e intitulado Imaginación y el Arte en la Infancia, reconoce dos impulsos en la actividad humana: uno reproductivo que requiere de la memoria y otro que moldea, crea y que tiene relación con la imaginación: "es precisamente la actividad creadora del hombre la que hace de él un ser proyectado hacia el futuro, un ser que contribuye a crear y que modifica su presente" (p. 3). Igualmente, manifiesta que la imaginación hace posible la creación artística y científica. Este sicólogo reconoce cinco formas que conectan la imaginación con la realidad: la acumulación de experiencia, el apoyo imaginario, la doble expresión de las emociones, el signo emocional común y la representación emocional de la realidad. Estas formas destacan que la memoria y la imaginación están presentes en toda acción humana. La imaginación posibilita la creación artística y científica y requiere de la memoria y de la experiencia vivida; esta segunda, a su vez, requiere de la imaginación. Imaginación, experiencia y emoción se apoyan mutuamente y se complementan en el acto humano, puesto que sentimiento y pensamiento mueven la creación humana (p. 9). Después añade que la fantasía se construye desde la memoria y la realidad, y por tanto depende de las experiencias vividas: "cuanto más rica sea la experiencia humana, tanto mayor será el material del que dispone esa imaginación" (p. 6). La fantasía no es meramente reproductiva, sino que fecunda nuevas formas de ver el mundo y está en las obras de arte, sobre todo en la literatura, cuya lógica fantástica influye en la conciencia social y nos ayuda a comprendernos (p. 10).

Respecto a la creencia, se la define como "parte del conocimiento, perteneciente al dominio cognitivo, compuesta por elementos afectivos, evaluativos y sociales" (Gómez, 2003, p. 234). Las creencias, según Gilbert (1991), son "concepciones o ideas, formadas a partir de la experiencia, sobre las matemáticas, su enseñanza y aprendizaje y sobre sí mismo en relación con la disciplina" (citado en Caballero y Blanco, 2007, p. 3).

Asítambién, los procesos educativos no dependen solo de los conocimientos disciplinares y académicos, sino que hay múltiples factores en la personalidad de quien aprende que intervienen en la eficacia de ese proceso. Esto implica la presencia de un profesor consciente de lo fundamental que es la educación emocional, capaz de comprender los estados emocionales de los estudiantes y "capaz de enseñar la aritmética del corazón y la gramática de las relaciones sociales" (Extremera y FernándezBerrocal, 2002, p. 374), dado que este profesor interfiere en el desarrollo de la personalidad de sus estudiantes. De allí que la preparación de todo profesional, especialmente los maestros, debe partir de la educación emocional (Fernández et al., 2009). Así también, Gardner (2016) sostiene la importancia de que las personas dedicadas al estudio de la ciencia hagan estudios humanísticos para evitar sufrir crisis y depresiones:

las ingenierías y estudios tecnológicos acaban dándote una sensación de control sobre tu vida en el fondo irreal: sólo te concentras en lo que tiene solución y en las preguntas con respuesta. Y durante años las hallas. Pero, cuando con la madurez descubres que en realidad es imposible controlarlo todo, te desorientas.

Así las cosas, si un maestro pretende formar a sus estudiantes más allá de la mera racionalidad debe esforzarse en la práctica de actitudes positivas y de profundo respeto, que 
contribuyan a la formación de capacidades, como "saber elogiar de manera sincera e incondicional, dar más importancia a la persona que a los problemas que tiene, poner por delante de la conducta las causas de ésta, manifestar interés e ilusión por el trabajo" (Fernández Domínguez, 2005, citado en Fernández et al., 2009, p. 43). Estas actitudes proyectan unas competencias socioafectivas necesarias para que el maestro adquiera la dimensión humana integral que se requiere en el aula, tales como la capacidad para expresar y controlar emociones, para resolver conflictos, tolerar la incertidumbre y tomar decisiones.

En este punto, y dada esta relación necesaria entre actitud y cognición en el proceso educativo, surge una interesante pregunta: ¿cuáles configuraciones debe tener la didáctica de la matemática si quieren incluir afectos, emociones y actitudes tendientes a formar la razonabilidad de los estudiantes de matemáticas? La respuesta, nada trivial, va desde la inclusión de la motivación en el aula hasta la posición estética asumida por el profesor en el ejercicio de la enseñanza; requiere de mediaciones tendientes a despertar la curiosidad, el asombro y las emociones de los estudiantes.

Es clave saber que la relación entre motivación y aprendizaje es relevante, puesto que el comportamiento de un estudiante al aprender depende lo que lo motiva a aprender (Skemp, 1980; Gómez, 2005; Alsina \& Domingo, 2007). La motivación intrínseca puede incrementar el rendimiento académico y favorecer el aprendizaje significativo. Skemp (1980) refiere

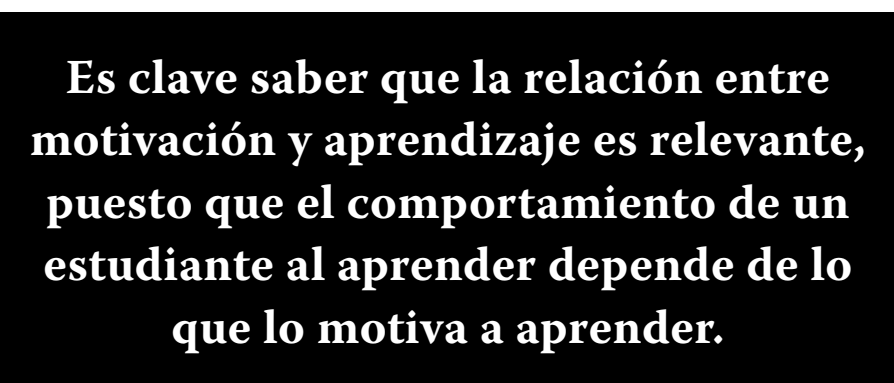

las motivaciones intrínsecas "como aquellas que surgen de dentro del sujeto, y que hacen que las matemáticas sean una actividad que recompensa en sí misma (citado en Alsina y Domingo, 2007, p. 24). Estos últimos elaboran una propuesta de aprendizaje activo, mediante el programa de transposición didáctica de los conceptos matemáticos de Domingo (2004), para mejorar el grado de motivación académica de los estudiantes por el aprendizaje de las matemáticas. Según ellos, "la percepción que el sujeto tiene del desarrollo de su rol como estudiante se correlaciona positivamente con el rendimiento académico, la aceptación de los compañeros, el liderazgo y la responsabilidad" (Alsina y Domingo, 2007, p. 27). La motivación de los estudiantes depende del conocimiento de sus necesidades y expectativas y del nivel comunicativo del profesor (Farias y Pérez, 2010). Así, "si el estudiante presenta un patrón motivacional negativo, frente a una dificultad, aumentará su ansiedad y hasta se angustiará pensando que la causa de la dificultad es su incapacidad y, por tanto, adoptará una actitud defensiva" (Font, 1994, p. 14). También acota que educar en matemáticas no es tan simple como explicar algoritmos y aplicar fórmulas sino que es la búsqueda de la emotividad, "que la gente se sienta feliz haciendo matemáticas, que le haga ilusión ir a clase, que se sepa transmitir la ilusión por el descubrimiento, por compartir lo que se está haciendo" (p. 8).

Es común saber que se aprende matemáticas para resolver los problemas de la cotidianidad, pero todavía más, para ser libres e independientes al tomar decisiones y para ser más felices al buscar el crecimiento espiritual y estético. La comprensión de un problema matemático que se quiere resolver está mediada por el dominio emocional de quien intenta resolverlo (Gómez Chacón, 2000; Estrada y Diez, 2011; Castelló et al., 2010; Gil et al., 2006).

Aunque el deseo de humanización de la enseñanza de la matemática se ha intensificado en los últimos tiempos, no se puede desconocer el interés puesto en la antigüedad por el sentido 
estético y la belleza de la forma en relación con la matemática (Boyer, 1994). Como tampoco puede ignorarse la vivencia de matemáticos como Poincaré, Hadamard y Halmos, quienes desarrollaron trabajos que evidencian el papel de los afectos y las actitudes en la actividad matemática, así como el carácter creativo de la misma. Si bien la comprensión de la matemática ha sido una preocupación de todos los tiempos, la educación de aspectos que rodean a quien aprende matemáticas también es materia de formación en el aula.

Además de alfabetizar con letras y números, es necesario alfabetizar en las emociones, las habilidades sociales, la toma de decisiones, el manejo de relaciones y en definitiva, en los problemas que afectan de verdad a nuestra vida, que pocas veces son las raíces cuadradas (aunque eso también sea necesario aprenderlo) (Feixas, 1999, citado en Teruel, 2000, p. 145).

Caballero y Blanco (2007) determinan que los factores afectivos de los estudiantes que aspiran a ser maestros dependen de los afectos de sus profesores. Los estudiantes consideran las matemáticas útiles y necesarias para desenvolverse socialmente y no las tildan de aburridas. En esta misma dirección, Gil, Blanco y Guerrero (2006) y Serrano (2011) sostienen que existe una relación bidireccional entre los afectos y el aprendizaje; esto es, aprender matemática eleva las emociones y estar bien emocionalmente permite aprender matemáticas.

Gómez Chacón (2003) introduce el término metaafecto para referirse a la conciencia de las emociones propias como observar, identificar y nombrar: "es estar atento a los estados internos sin reaccionar ante ellos y sin juzgarlos. Ser consciente de uno mismo significa ser consciente de nuestros estados de ánimo, y de los pensamientos que tenemos acerca de esos estados de ánimo" (p. 230). Para ella, todas las creencias que tienen los estudiantes modifican sus comportamientos en el estudio de la matemática, y este sistema de creencias

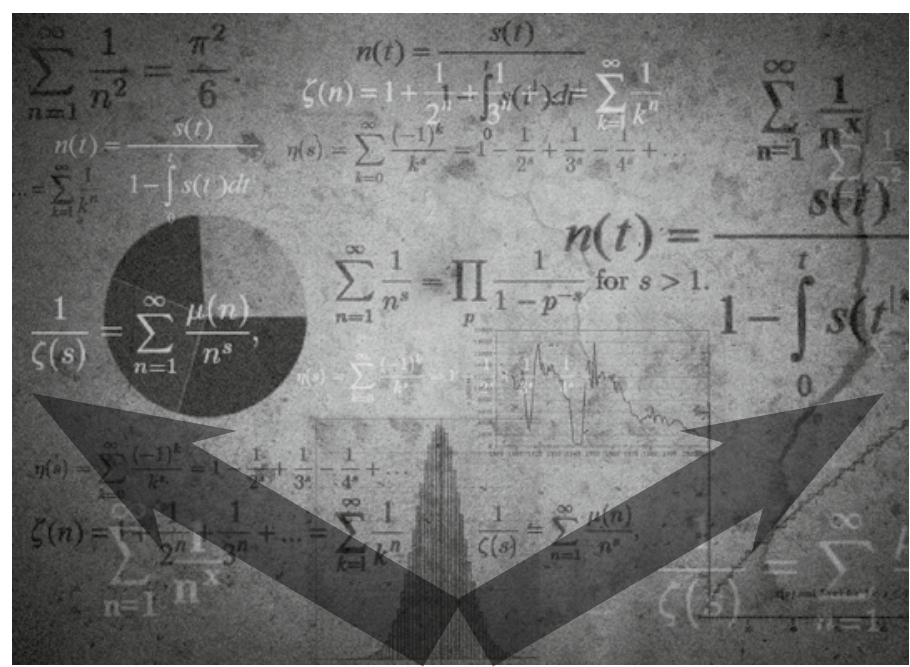

depende de la intervención de los profesores en el aula.

Así mismo, Gómez Chacón (2009) distingue entre actitudes hacia la matemática y actitudes matemáticas. Las primeras, según esta autora, se refieren "a la valoración y al aprecio de esta disciplina y al interés por esta materia y por su aprendizaje, y subrayan más el componente afectivo que el cognitivo; aquélla se manifiesta en términos de interés, satisfacción, curiosidad, valoración, etcétera" (p. 11); mientras que las actitudes matemáticas inoculan estrategias cognitivas como "la flexibilidad de pensamiento, la apertura mental, el espíritu crítico, la objetividad, etc." Ambas se forman en el paso por la escuela donde se pueden tener capacidades, pero no actitudes, y viceversa.

También Rodríguez (2011) propone la formación de un ser humano crítico, pensante y transformador de la realidad mediante la educación matemática humanista desde la mirada de la pedagogía integral. Y esto, según la doctora Rodríguez, requiere de una matemática viva que tenga en cuenta las vivencias, el contexto, los sentires y los diálogos interiores de los estudiantes, así como la relación dialógica entre estudiante, maestro y comunidad. La educación integral es "un encuentro dialógico entre profesores, estudiantes, comunidades; es un proceso cargado de: subjetividad, 
intencionalidad, transcendencia, incertidumbre, necesidades motivaciones, proyectos que no pueden ser apartados a menos que se destruya la misma educación” (p. 101).

Todo esto se logra si se mira la educación como un encuentro humano que involucre la experiencia, las emociones y los sentimientos; cuestión compleja, puesto que la matemática ha sido considerada como una ciencia árida, rigurosa y objetiva, y no se ha reconocido como una construcción humana relacionada con las necesidades sociales que enseña a razonar (Castelló et al., 2010). Aunque, por el contrario, los trabajos de Schoenfeld (1992) sostienen que las creencias que tengan, tanto el profesor como los estudiantes, inciden notablemente en la capacidad para resolver un problema.

Ahora, la educación emocional que se requiere en la clase de matemáticas puede darse con la mediación del arte, de la historia y de la literatura. Novelas como La soledad de los números primos, El teorema del loro, En busca de Klingsor o El curioso incidente del perro a medianoche, entre otras, además de múltiples relatos que motivan al estudiante, le ayudan al docente a "despertar la curiosidad, estimular la imaginación del alumno y ofrecer oportunidades para el desarrollo de creatividad" (Bazán y Aparicio, 2006, p. 7).

Se requiere una enseñanza que supere el abuso de contenidos y se concentre en procesos de pensamiento, sin olvidar que la razón no ha de separarse de lo estético, y, en este orden de ideas, la razonabilidad no impide la exploración profunda de lo humano que somos en un momento de la clase. Para ello necesitamos volver al amor como proceso evolutivo y propiciar el conocimiento de la matemática paralelo a la educación emocional en una correspondencia mutua entre la mente que piensa y la mente que siente; el conocimiento matemático eleva amor propio y el amor propio potencia el entendimiento de la matemática.
La didáctica de la matemática tiene que ocuparse de la relación entre el estado emocional del estudiante y la actitud que tiene hacia la matemática, lo que condiciona el aprendizaje de la misma, dado que es fundamental una actitud positiva hacia la matemática. Para esto se tiene que pensar en la formación de un maestro que se piense líder emocional de la clase (Extremera \& Fernández Berrocal, 2001, p. 375) y que utilice mediaciones positivas que le permitan comprender que "el reto del educador/a es irrumpir e interrumpir los sentimientos negativos como paso previo a la necesaria reconstrucción afectiva/cognitiva que deben tener lugar para el avance del estudiante encontrando caminos didácticos que favorezcan estos aspectos" (Gómez, 2000, p. 154). Más aún, hace falta un maestro que comprenda que el acto de enseñar exige, además de preparación científica, preparación física y emocional; que sepa que enseña con "los sentimientos, con las emociones, con los deseos, con los miedos, con las dudas, con la pasión y también con la razón crítica" (Freire, 1998, p. 8); puesto que "enseñar es una tarea profesional que exige amorosidad, creatividad, competencia científica" (p. 9).

Si bien el afecto es una asignatura pendiente (Beltrán, 1996, p. 401), en la educación matemática es todavía más lejana la posibilidad de incluir las emociones, los afectos y las actitudes. Henao Ciro (2010, 2012, 2014) ha sostenido la necesidad de humanizar la enseñanza de la matemática desde la literatura, al convocar los sentidos de los profesores de matemática para acercar más esta ciencia a la vida y crear un diálogo necesario entre el arte y la matemática para no tener que preguntarse "¿qué importa que el entendimiento se adelante, si el corazón se queda?" (Gracián, 2001, p. 36); puesto que razón y emoción habitan un espacio posible en el aula en la medida que el aprendizaje no ignora las emociones, sino que, como galleta cubierta de chocolate, el aprendizaje se envuelve en ellas (Gardner, 2000, p. 89). 


\section{Referencias}

Alsina, A. y Domingo, M. (2007). Cómo aumentar la motivación para aprender matemáticas. Suma, (56), pp. 23-31. Recuperado de http:// revistasuma.es/IMG/pdf/56/023-031.pdf

Bazán, J. \& Aparicio, A. (2006). Las actitudes hacia la Matemática-Estadística dentro de un modelo de aprendizaje. Educación, 15 (28), pp. 7-20. Recuperado de http://revistas.pucp. edu. pe/index.php/educacion

Beltrán, J. \& Pérez, L- (1996). Inteligencia, pensamiento crítico y pensamiento creativo. En Beltrán, J. \& Genovard, C. (Eds.): Psicología de la instrucción I. Variables y procesos, pp. 429-503, Madrid: Síntesis.

Bisquerra, R. (2005). La educación emocional en la formación del profesorado. Revista interuniversitaria de formación del profesorado, 19 (3), pp. 95-114. Recuperado de http://www.redalyc. org/pdf/274/27411927006.pdf

Blanco, L. y Guerrero, E. (2002). Profesionales de las Matemáticas y Psicopedagogos. Un encuentro necesario. En: Penalva, G. Torregosa y J. Valls (Coords.), Aportaciones de la Didáctica de la matemática a diferentes perfiles profesionales (pp. 121-140). Actas del V Simposio de Didáctica de las matemáticas. Universidad de Alicante.

Boyer, C. (1994). Historia de la Matemática. Madrid: Alianza Editorial.

Bueno, C., Teruel, M. y Valero, A. (2005). La inteligencia emocional en alumnos de magisterio: la percepción y comprensión de los sentimientos y las emociones. Revista interuniversitaria de formación del profesorado, 19 (3), pp. 169194. Recuperado de http://www.redalyc.org / pdf/274/27411927010.pdf

Caballero, A. y Blanco, L. (2007). Las actitudes y emociones ante las Matemáticas de los estudiantes para Maestros de la Facultad de Educación de la Universidad de Extremadura. Recuperado de http://www.eweb.unex.es/eweb/liblanco/ documentos/anacaba.pdf

Castelló, M, Codina, R. y López P. (2010). Cambiar las actitudes hacia las matemáticas resolviendo problemas. Una experiencia en
Formación del Profesorado de Educación Primaria. Unión (22), pp. 65-76.

Estrada, A \& Diez, J. (2011). Las actitudes hacia las Matemáticas. Análisis descriptivo de un estudio de caso exploratorio centrado en la Educación Matemática de familiares. Recuperado de http://webs.uvigo.es/reined/

Extremera, N. \& Fernández-Berrocal, P. (2003). La inteligencia emocional en el contexto educativo: hallazgos cientificos de sus efectos en el aula. Educación, 332, pp. 97-116. Recuperado de www.mecd.gob.es/dctm/revistade- educacion/articulosre332/re3320611443.pdf ?documentId=0901e72b812 56ae3

Farias, D \& Pérez, J (2010). Motivación en la Enseñanza de las Matemáticas y la Administración. Formación Universitaria, 3 (6), pp. 33-40. Recuperado de http://www.scielo.cl/pdf/ formuniv/v3n6/art05.pdf

Fernández, M. (2005). Más allá de la educación emocional. La formación para el crecimiento y desarrollo personal del profesorado. PRH como modelo de referencia. Revista interuniversitaria de formación del profesorado, 19 (3), pp. 195-251. Recuperado de www.redalyc.org/ pdf/274/27411927011.pdf

Fernández, M., Palomero, J. \& Teruel, M. (2009). El desarrollo socioafectivo en la formación inicial de los maestros. Suma, (17), pp. 10-16. Recuperado de http://www.aufop.com

Font, V. (1994). Motivación y dificultades de aprendizaje en matemáticas. Suma, 17, pp. 10-16. Recuperado de http://revistasuma.es/ IMG/pdf/17/010-016.pdf

Freire, P. (1998). Cartas a quien pretende enseñar. México: Siglo Veintiuno.

García, J. (2012). La educación emocional, su importancia en el proceso de aprendizaje. Educación 36 (1), pp. 1-24. Recuperado de http:// dcb.fi-c.unam.mx/Eventos/Foro4/Memorias/ Po nencia_100.pdf

Gardner, H. (2000). La educación de la mente y el conocimiento de las disciplinas. Barcelona: Paidós. 
Gardner, H. (2016). Una mala persona no llega nunca a ser un buen profesional. Recuperado de http://www.lavanguardia.com/lacontra/2016 0411/401021583313/una-mala-persona-nollega-nunca-a-ser-buen-profesional.html

Gil, N., Guerrero, E. \& Blanco, L. (2006). El dominio afectivo en el aprendizaje de las matemáticas. Electronic Journal of Research in Educational Psychology, 4 (8), pp. 47-72. Recuperado de www. redalyc.org/articulo.oa?id=293123488003

Gil, N; Blanco, L \& Guerrero E. (2006). El papel de la afectividad en la resolución de problemas matemáticos. Educación, (340), pp. 551-569. Recuperado de www.revistaeducacion.mec.es / re340/re340_20.pdf

Gómez, I. (2000). Matemática emocional. Los afectos en el aprendizaje matemático. Madrid: Narcea.

Gómez, I. (2003). La tarea intelectual en matemáticas; afecto, meta-afecto y los sistemas de creencias. Recuperado de www.emis.de/journals/ BAMV/conten/vol10/igomez.pdf

Gómez, I. (2005). Motivar a los alumnos de secundaria para hacer matemáticas. Recuperado de http://www.mat.ucm.es/ imgomezc/almacen/ pisa-motivar

Gómez, I. (2009). Actitudes matemáticas: propuestas para la transición del bachillerato a la universidad. Educación Matemática, 21 (3), pp. 5-32.

Gracián, B. (2001). El héroe. Barcelona: Estrategia Local.

Henao Ciro, R. (2010). Peirce y la representación matemática. Recuperado de http://www.unav.es/ gep/ArticulosOnLineEspanol.html.

Henao Ciro, R. (2012). Un teorema literario y otros ensayos de interés en educación matemática. Madrid: Editorial Académica Española.

Henao Ciro, R. (2014). La razonabilidad estética como proceso interhumano y abductivo desde "Un descenso al Maelstron". Enunciación, 19(1), pp. 49-60.

Henao Ciro, R. \& Moreno, M. (2015). Aproximación histórica al concepto de lógica: avances parciales de una investigación que promueve la experiencia estética en maestros en formación en Matemática y Literatura. Ikala, 20(2), pp. 233-244.

Hidalgo, S. et al. (2008). Estudio longitudinal del componente emocional matemático en el paso de primaria a secundaria. Recuperado de www. seiem.es/publicaciones/archivos publicaciones/ comunicacionesgrupos/GruposXII/Didacticade1Analisis/HidalgoMarotoOrtegaYPalacios.pdf

Mcleod, D. (1994). Research on affect and mathematics learning in the JRME: 1970 to the present. Journal for Research in Mathe matics Education, 25(6), 637-647. Recuperado de https://www.jstor.org/stable/749576? seq=1\# page_scan_tab_contents

Rodríguez, M. (2011). Pedagogía integral, humanización y educación matemática: una mirada y un horizonte para construir una educación matemática humanista. Diálogos Educativos, 21, pp. 98-109. Recuperado de http://www.umce. cl/ dialogos/n21_2011/ rodriguez.swf

Schoenfeld, A. (1992). Learning to think mathematically: Problem solving, metacognition, and sense-making in mathematics. Recuperado de http://jwilson.coe.uga.edu/EMAT7050/ Schoenfeld_MathThinking.pdf

Serrano, A. (2011). Procesos metafectivos en el aprendizaje de las matemáticas. (Tesis de Maestría) Universidad Autónoma de Manizales. Recuperado de http://repositorio.autonoma.edu. co /jspui/bitstream/11182/182/1/procesos $\% 20$ metafectivos\%20en\%20el\%20aprendizaje $\% 20$ de\%20las\%20matematicas\%20Sept\%202011.pdf

Teruel, M. (2000). La inteligencia emocional en el currículo de la formación inicial de maestros. Recuperado de http://www.aufop.com/aufop/ uploaded_files/articulos/1223493322.pdf

Vigotsky, L. (2004). Teoría de las emociones. Madrid: Ediciones Akal.

Vigotsky, L. (1983). La imaginación y el arte en la infancia. Ensayo psicológico. Recuperado de http://moodle2.unid.edu.mx/dts_cursos_mdl/ lic/ED/DC/AM/10/

Vigotsky, L. (1982) Imaginación y el arte en la infancia (ensayo psicológico). Bs. As.: Akal. 